\title{
Reflections of Essence
}

\author{
Steve DiPaola \\ Simon Fraser University \\ 888 University Dr. Burnaby BC \\ Canada V5A1S6 \\ sdipaola@sfu.ca
}

\author{
Sara Salevati \\ Simon Fraser University \\ 888 University Dr. Burnaby BC \\ Canada V5A1S6 \\ sara_salevati@sfu.ca
}

\section{INTRODUCTION}

This piece takes a known traditional art form, fine art portrait painting - its history, style, palette, and knowledge space - and disrupts it into an experience, an emotion and artificial intelligence interactive. We use our Creative Artificial Intelligence System (CAIS) software tools (we write) that take as source a photograph of a interacting viewer in front of our digital canvas and through their selection of an emotion (how they feel or what the effect is that they want to explore) creates and evolves an art portrait of them (Figure $1 \& 2)$.

This photograph as source is evolved into a final artwork that uses the rules, styles and elements from our creativity based Al portrait painting system and maps different texture, brush stroke and palette to the various emotions based on survey studies. We want to focus on the emotional journey while interacting with a generative art piece and involving our users/viewer in the meaning making process. This spiral of experiencing, reflecting and making provides a participatory platform for cocreation of ideas, which is visitor focused, openended and prepares the viewer for broader, richer, learning experiences.

\section{INTERACTIVE EXPERIENCE}

We intermingle within the disruptive themes of GENERATIVE ART (the system), BODY, EMBODY + PERFORM (interacting presence, façade and emotion, group interaction) and SCIENCE + INTERDISCIPLINARITY. Our generative software that looks deeply (academic with studies with eye tracking studies, emotion studies, texture and color synthesis software, ...) at the 2000 year old practice of fine art portrait painting and uses art styles, palette and other factors from a known traditional and art generated art work, an artist's body of work, and art in general to produce in software emotional user interactive controls. We have several published papers on this type of analysis in our past work of Rembrandt (DiPaola, et al, 2010), Picasso (DiPaola, Smith 2013) and Boccioni (DiPaola, Salevati, 2014).

In this way, we do not want to reduce the viewer's experience into a few variables or simple templates of styles. But instead use the more open and human sense of emotion as the core of what humans are and how we experience the world. All aspects of the final images from our interactive are created generatively with texture synthesis, cognitive synthesis (using the palette and rules of portrait painting algorithms) as well as results validated from our emotional mapping survey studies.

\section{REFERENCES}

DiPaola, S. \& Salevati, S. (2014) Using a Creative Evolutionary System for Experiencing the Art of Futurism. International Conference on Electronic Imaging \& the Visual Arts (EVA 2014), Florence, Italy, pp. 88-93.

DiPaola, S. \& Smith, A. (2013) Interactively Exploring Picasso's Multi-dimensional Creative Process in Producing Guernica. Electronic Visualisation and the Arts (EVA 2013), London, UK, pp. 25-31. British Computer Society, London.

DiPaola, S., Riebe, C., \& Enns J. (2010) Rembrandt's Textural Agency: A Shared Perspective in Visual Art and Science. Leonardo, 43(3):145-151. 

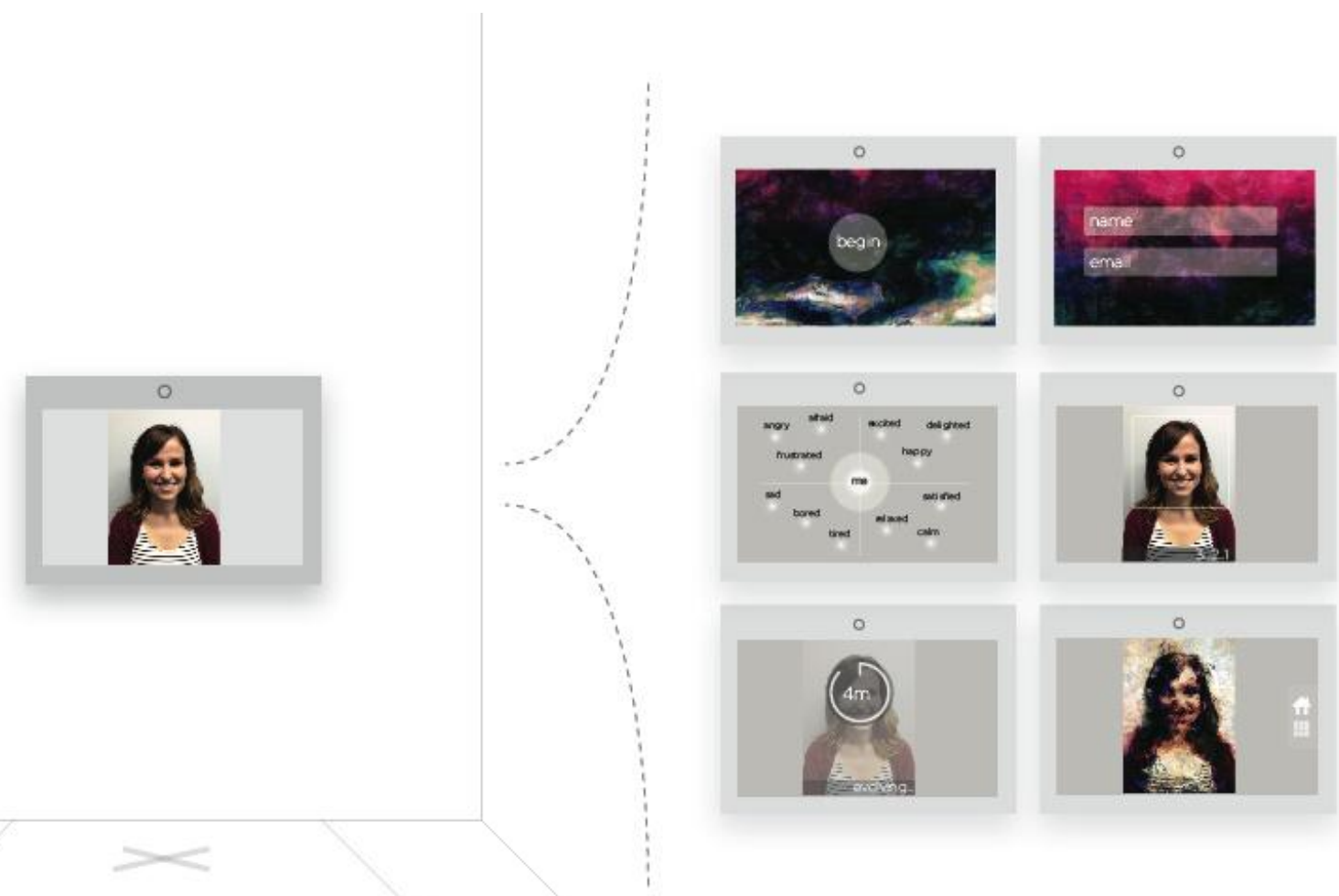

Figure 1: This is a depiction of the system in process, participants begin by entering their name and email, then they select an emotion that they want to explore, a photo of the viewer is then taken, processing occurs and a portrait style is made based on emotional input;

we are investigating (as options) using the user's mobile device as well as biosensing emotion
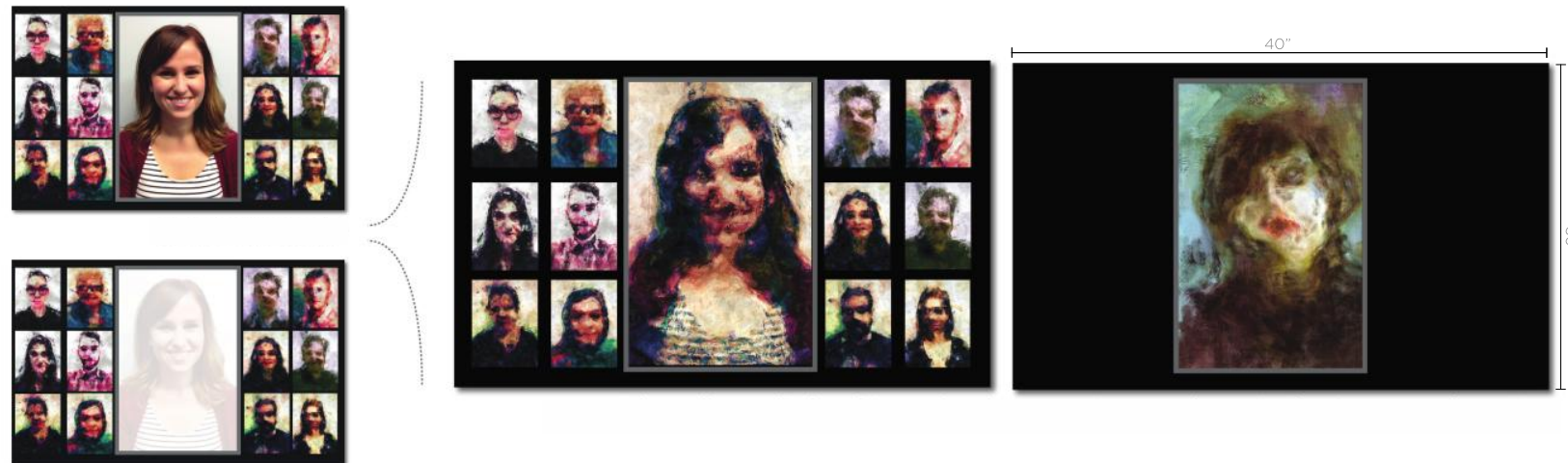

Figure 2: This demonstrates the instillation on 2 large screens (40" $\times 30$ ") within the exhibition; Screen1 displays the participant's interaction as they explore the system, their photo takes the main frame amongst the past portraits and evolves into a portrait style;

it will remain in the large frame until another participant begins to interact with our system; Screen 2 displays a pre-made movie showing range of emotions of our past work

to engage the audience while they wait for their portraits to evolve 